\title{
The Role of Minimally Invasive Glaucoma Surgery Devices in the Management of Glaucoma
}

\author{
Murray Fingeret, OD, FAAO ${ }^{1,2 *}$ and Jaime E. Dickerson, Jr, $\mathrm{PhD}^{3,4}$
}

\begin{abstract}
SIGNIFICANCE: Noncompliance is a problem affecting glaucoma patients. Approaches to improve adherence include the use of drug-delivery systems and safer forms of surgery. Minimally invasive glaucoma surgery (MIGS) has reduced complications, particularly in combination with cataract surgery, and with its good intraocular pressure (IOP) reduction may reduce or eliminate glaucoma medications.

Glaucoma is a progressive disease and a leading cause of irreversible blindness. Elevated IOP is the most important risk factor, but effective medical management is dependent on patient adherence. This review summarizes the adherence problem in glaucoma and the efforts, including MIGS, to provide effective IOP control that is not dependent on patient compliance.

The current understanding of patient adherence to pharmacological treatment of glaucoma is discussed including the challenges facing glaucoma patients. Historical approaches to providing IOP control in a sustained and reliable way are presented culminating in a review of the burgeoning use of MIGS devices.

It is estimated that, in the United States, $27 \%$ of prescriptions written, across all medications, are not filled or are filled but not taken. For ocular hypotensive medications, even when filled, a large percentage (which varies widely by study) are not instilled as prescribed. To address this problem, methods for sustained drug delivery have been and continue to be developed, as well as surgical and laser approaches. Most recently, MIGS devices have gained popularity because of the ease of implantation during cataract surgery, favorable safety profile, and the possibility for effective and long-lasting IOP lowering, as well as the reduction or elimination of need for IOP-lowering medication. Poor adherence to treatment is relatively common among glaucoma patients and is associated with progression of disease. Recommending MIGS implantation during cataract surgery may offer optometrists a valuable treatment option in managing glaucoma patients, particularly where good adherence is in doubt.
\end{abstract}

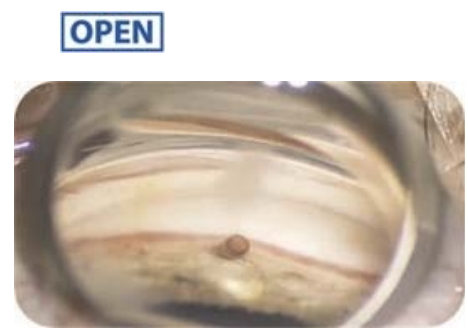

\begin{abstract}
Author Affiliations:
${ }^{1}$ New York Harbor Health Care System, Department of Veterans Affairs, Brooklyn, New York

${ }^{2}$ State University of New York College of Optometry, New York, New York ${ }^{3}$ Alcon Research, Ltd., Fort Worth, Texas ${ }^{4}$ University of North Texas Health Science Center, Fort Worth, Texas *murray.fingeret@va.gov
\end{abstract}

Optom Vis Sci 2018;95:155-162. doi:10.1097/OPX.0000000000001173

Copyright (C) 2018 The Author(s). Published by Wolters Kluwer Health, Inc. on behalf of the American Academy of Optometry. This is an open-access article distributed under the terms of the Creative Commons Attribution-Non Commercial-No Derivatives License 4.0 (CCBY-NC-ND), where it is permissible to download and share the work provided it is properly cited. The work cannot be changed in any way or used commercially without permission from the journal.

Glaucoma is the leading cause of irreversible blindness worldwide and is a silent, progressive disease. The global prevalence of glaucoma is estimated to be 76 million by $2020 .{ }^{1}$ The general lack of symptoms (for open-angle glaucoma) and the fact that significant loss of retinal ganglion cells may occur before glaucomatous visual field changes are detected underscore the need for early detection and consistent, effective treatment. ${ }^{2,3}$ Elevated intraocular pressure is the primary risk factor for glaucoma, and treatment for glaucoma is directed at lowering intraocular pressure either medically or surgically. ${ }^{4}$ The Ocular Hypertension Treatment Study showed that reduction of intraocular pressure by an average of $22.5 \%$ through medical intervention decreased conversion from ocular hypertension to glaucoma over a 5-year period to approximately half that of untreated controls (4.4 vs. 9.5\%). ${ }^{5}$ Similarly, the Early Manifest Glaucoma Trial showed that treatment of newly diagnosed primary open-angle glaucoma with argon laser trabeculoplasty plus betaxolol reduced the risk of disease progression at 6 years to half of that for untreated controls (hazard ratio, 0.50; 95\% confidence interval, 0.35-0.71). ${ }^{6}$ Moreover, the risk of progression was decreased by approximately $10 \%$ for each $\mathrm{mmHg}$ intraocular pressure was reduced from baseline. ${ }^{6}$ In the Advanced Glaucoma Intervention Study, for patients with more advanced disease, maintenance of intraocular pressure such that $100 \%$ of measurements over the 7-year follow-up were less than $18 \mathrm{mmHg}$ resulted in complete absence of visual field deterioration. ${ }^{7}$ Preservation of vision in patients with glaucoma is clearly dependent on control of intraocular pressure. With medical therapy generally the first line of treatment, successful control of intraocular pressure is largely dependent on patient adherence. ${ }^{8-10}$

The optometrist's role in the care of the glaucoma patient has expanded since 1975 from detection and referral to treatment and management with pharmaceutical agents and, in some states, laser procedures. Optometrists are often the first to diagnose glaucoma and are licensed to treat glaucoma medically in 49 of the states in the United States and Canada. The American Optometric Association guidelines for the treatment and management of glaucoma stress the importance of "continuity of care, compliance with therapy, communication with patients' physicians, and possibly comanagement with a glaucoma specialist." ${ }^{11}$ When medical treatment fails, or when adherence to medical therapy is inadequate, patients are referred to ophthalmologists for surgical intervention. ${ }^{12}$ Since the publication of these guidelines in 2011 , a new modality for the management of mild to moderate glaucoma has emerged. ${ }^{13}$ Minimally (or micro) invasive glaucoma surgery provides an alternative to more invasive surgical methods or to laser procedures. These can generally be done along with cataract surgery minimizing additional risk or, in some jurisdictions, as standalone procedures. Several studies have demonstrated these devices to provide long-term intraocular pressure lowering and the potential to reduce or eliminate the need for medications in some patients. ${ }^{13,14}$ This review summarizes the patient adherence problem in glaucoma and reviews the efforts, including minimally invasive glaucoma 
surgery, to provide effective intraocular pressure control that is not dependent on patient compliance.

\section{BARRIERS TO ADHERENCE}

The problem of adherence and persistence with medications affects virtually all areas of medicine. Lack of adherence is estimated to cause approximately 125,000 deaths and at least $10 \%$ of hospitalizations, with costs to the U.S. healthcare system between $\$ 100$ billion and $\$ 289$ billion a year. ${ }^{15}$ C. Everett Koop's (perhaps apocryphal) statement "Drugs don't work in patients who don't take them" has particular resonance for a silent, progressive, and potentially blinding disease such as glaucoma. In a decade-old systematic review of the literature summarizing 29 studies, Olthoff et al. ${ }^{16}$ concluded that nonadherence among glaucoma patients was commonplace. The proportion of patients deviating from the prescribed regimen was variable but reached as high as $80 \%$. Given the potential consequences of unchecked glaucoma progression, such a high prevalence of nonadherence may seem surprising. It is important to remember, however, that glaucoma, especially open-angle glaucoma, is often asymptomatic, even when there are functional deficits sufficient to result in falls and motor vehicle accidents. ${ }^{17}$ This lack of symptoms and the slow disease progression make it difficult for patients to perceive a worsening in their condition and thus heighten their level of concern. In addition, the glaucomatous population tends to be skewed toward the older demographic with a prevalence three to eight times higher in the oldest age groups when compared with people in their 40 s. $^{18}$ Several studies have shown that most patients contend with multiple barriers. These include skepticism that glaucoma will cause vision loss, skepticism that glaucoma medications will prevent vision loss, decreased self-efficacy, lack of knowledge about glaucoma, physician mistrust, difficulty administering eye drops, medication costs, medication adverse effects, forgetfulness, difficulties with complex medication schedules, and life stress. ${ }^{19-26}$ The disproportionate affliction (of glaucoma) in older individuals introduces age-specific challenges; generally, age-related comorbidities that may affect the ability to self-administer drops include arthritis, palsy of various etiologies, Parkinson disease, and low vision. Any of these can create a significant barrier to correct usage of prescribed ocular hypotensive medications. In a study where the mean age was 66 years, Newman-Casey et al. ${ }^{24}$ reported that among adherent subjects approximately $50 \%$ cited their chief barriers to adherence were skepticism that glaucoma would lead to vision loss and skepticism that glaucoma medications would prevent vision loss. These barriers were also important to a similar proportion of nonadherent patients, but in addition, difficulty with instillation of eye drops, forgetfulness, poor self-efficacy, and difficulties with the medication schedule were frequent problems. ${ }^{24}$ Another study showed that approximately only one-third of glaucoma patients were able to successfully instill a single drop of medication. ${ }^{25}$ While evidence directly linking poor adherence to treatment with glaucoma progression is scant, it has been shown that worse adherence measured over a 3-month study period was associated with worse visual field defects. ${ }^{16,26}$

\section{IMPROVING ADHERENCE}

Recognizing that nonadherence with medical glaucoma therapy is a serious problem affecting a large proportion of glaucoma patients, clinicians, pharmaceutical companies, and device companies have taken multiple approaches in attempts to improve adherence (Fig. 1). The most successful to date has been the development of fixed combinations of ocular hypotensive drugs. ${ }^{27}$

While there are historic combination products with pilocarpine as a component, only three fixed combinations are available in the United States: dorzolamide 2\%-timolol 0.5\% (Cosopt [Akorn Pharmaceuticals, Lake Forest, IL] or generic), brimonidine $0.2 \%$-timolol $0.5 \%$ (Combigan [Allergan, Irvine, CA]), and brinzolamide 1\%-brimonidine $0.2 \%$ (Simbrinza [Alcon Laboratories, Fort Worth, TX]). Other fixed combinations incorporating a prostaglandin analog are available outside the United States. In a randomized controlled trial comparing adherence to the assigned treatment (travoprost/timolol fixed combination or travoprost and timolol in separate containers), Barnebey and Robin $^{28}$ found that significantly more patients were at least $80 \%$ adherent with the fixed combination compared with the

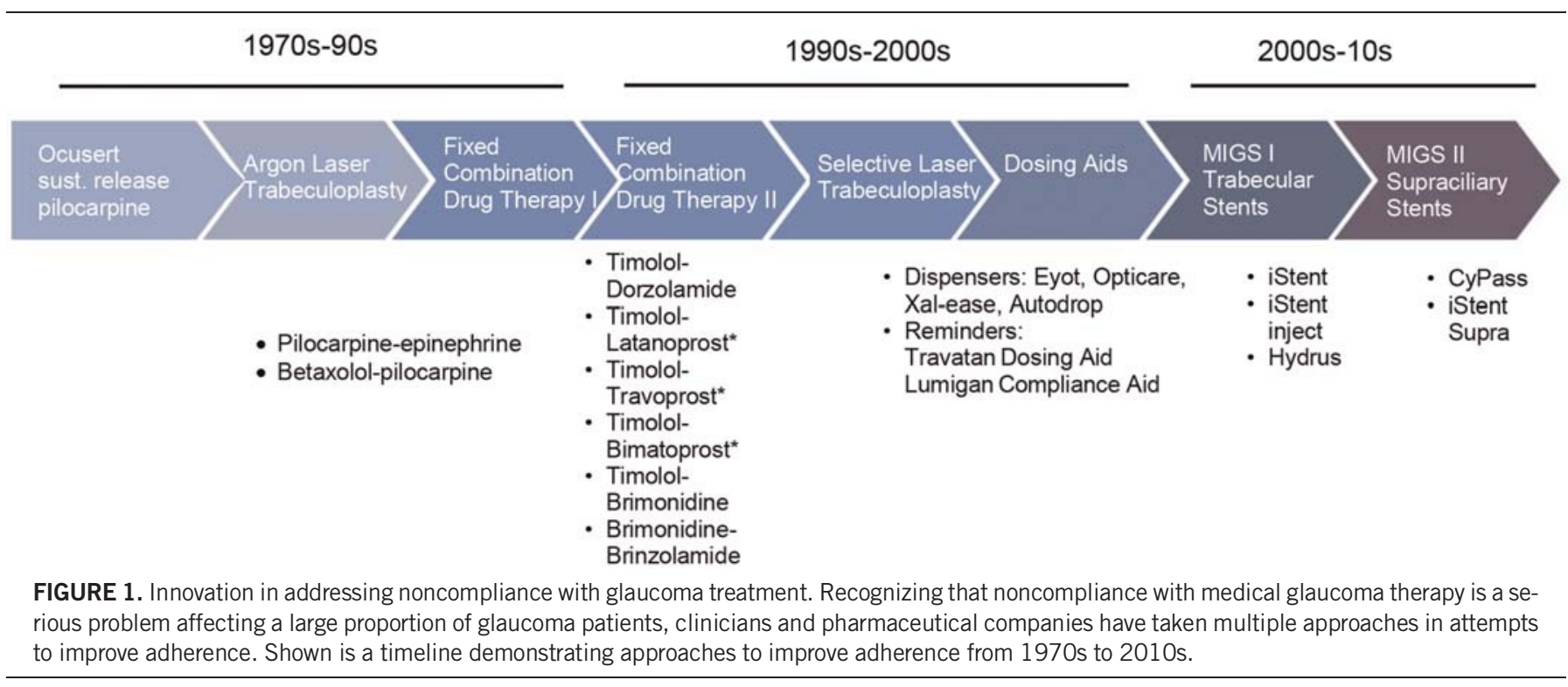


unfixed combination. However, even with the fixed combination, adherence decreased with time, and average adherence was only $60 \%$ of days through 12 months. Clearly, as noted above, there are other adherence barriers not addressed by regimen simplification.

Efforts have also been made to monitor and document patient adherence, both for research purposes and to provide clinicians and patients with objective, nonjudgmental data as a basis for discussion. The MEMS (medication event monitoring system), which time stamped the joint occurrence of dropper bottle cap removal and inversion, was an early such device. ${ }^{29}$ To address the problem of forgetfulness, dosing aids with visual and audible alarms that provide reminders to patients have been piloted (e.g., Travatan Dosing Aid [Alcon Laboratories]). Although adherence was shown to improve $(87.9 \%$ with alarm vs. $79.7 \%$ without alarm, $P=.02)$, it was mainly incremental. ${ }^{30}$ As tools to manage treatment, the dosing record retained by these devices does provide the clinician with useful information on patient adherence. Perhaps due to cost, these devices have not been widely used.

\section{SUSTAINED MEDICATION RELEASE}

Because glaucoma patients are largely responsible for managing their own ocular medication regimens, it may be that nonadherence is a problem that cannot be easily overcome. One potential answer would be to obviate the need for adherence through the use of some form of drug depot or device providing sustained release. ${ }^{31,32}$ Although this is not a new idea, few such products have been successfully developed. The Ocusert, which provided sustained release of a low level of pilocarpine into the tear film for 1 week, is no longer marketed, mainly due to the development of newer drugs requiring less frequent administration and also because of difficult (particularly for older patients) insertion. ${ }^{33}$ Currently in clinical development are prostaglandin-eluting devices to be placed in the fornices (bimatoprost), puncta (latanoprost), or canaliculi (travoprost); of these, only the intracanalicular travoprost appears to have advanced to phase 3 testing. One challenge in the development of continuous prostaglandin elution is that greater than once-daily dosing is often associated with diminished efficacy, possibly due to receptor sensitization. ${ }^{34}$ A device capable of timed, intermittent release could overcome this obstacle.

Another approach to sustained drug release is through injectable depots. Depots may be placed externally as in the sub-Tenon space or intraocularly in the anterior chamber. ${ }^{35,36}$ Bimatoprost (bimatoprost SR [Allergan]) and travoprost (ENV515; Envisia Therapeutics, Durham, NC) biodegradable implants injected intracamerally are currently in phase 2 (ENV515) or phase 3 (bimatoprost SR) development. Both of these aim to provide up to 6 months of intraocular pressure-lowering efficacy from a single injection. Results from early studies show intraocular pressure-lowering efficacy similar to topical dosing of the same drugs. ${ }^{36}$ The iDose device is a titanium travoprost-eluting implant injected through a clear corneal incision and secured in the anterior chamber. The device, which would be removed and replaced when depleted, is currently in phase 2 trial evaluating two different elution rates.

\section{LASER TRABECULOPLASTY}

For select patients, laser procedures are considered as initial therapy. This is particularly true where patients either are not tolerant of topical medications or cannot instill them, or when there is a high risk of nonadherence. ${ }^{11,37}$

Selective laser trabeculoplasty using a Q-switched, frequencydoubled Nd-YAG laser has been the method of choice since it was commercially available in 2001. The low-energy (relative to argon laser) laser specifically targets pigmented cells minimizing collateral damage. The intraocular pressure-lowering efficacy is similar to the older argon laser trabeculoplasty; however, at least for a second treatment, repeat selective laser trabeculoplasty shows efficacy close to the initial treatment. ${ }^{38,39}$ The efficacy of additional treatments beyond a second application has not been demonstrated, so the position of selective laser trabeculoplasty as either a stopgap or a true treatment platform that could provide patients with intraocular pressure control over many years or even decades is now not known.

Selective laser trabeculoplasty is a safe, office-based procedure with minimal adverse effects that include mild anterior chamber inflammation and transient intraocular pressure spikes, which are generally prevented or minimized with prophylactic preprocedure intraocular pressure-lowering medication. ${ }^{40,41}$ The main disadvantages of selective laser trabeculoplasty are that its intraocular pressure-lowering effect diminishes with time and the open question around efficacy of multiple repeat treatments. ${ }^{42,43}$ However, its potential to delay more invasive procedures by up to several years contributes to its overall utility.

\section{MINIMALLY INVASIVE GLAUCOMA SURGERY}

Minimally (or micro) invasive glaucoma surgery is a new and rapidly expanding category of glaucoma treatment. The definition of minimally invasive glaucoma surgery can vary and may include $a b$ externo approaches with little or no scleral dissection and little or no conjunctival manipulation. ${ }^{44}$ For the purposes of this discussion, minimally invasive glaucoma surgery devices are devices that use an ab interno microincisional approach, create minimal trauma to and disruption of normal anatomy and physiology, have a high level of biocompatibility, show effective intraocular pressure lowering, and have a very good safety profile with rapid recovery (Fig. 2, Table 1). ${ }^{51}$ In addition, for optimal utility, implantation of minimally invasive glaucoma surgery devices should be straightforward, rapid, relatively easy, appropriate for both glaucoma specialists and general ophthalmologists, and easily incorporated into other procedures. ${ }^{52}$

Minimally invasive glaucoma surgeries fill a gap that has existed in the treatment algorithm for glaucoma between medical therapy and laser at one end of the spectrum and traditional filtering glaucoma surgeries at the other. Before the advent of minimally invasive glaucoma surgery, a patient who had failed laser treatment and was not adequately controlled on medical therapy was referred for a trabeculectomy procedure. The intraocular pressure-lowering efficacy provided by trabeculectomy comes with known risks of intraoperative (e.g., torn conjunctiva, hemorrhage, choroidal effusion), postoperative (e.g., hypotony, failing or encapsulated bleb, uveitis, endophthalmitis, hyphema), and late postoperative (e.g., leaking bleb, blebitis, cataract) complications. ${ }^{4,53}$ In situations where intraocular pressure reduction goals are more modest, the glaucoma is newly diagnosed, the optic nerve head damage is only mild to moderate, and/or the medication burden creates the risk of poor adherence, minimally invasive glaucoma surgery is a treatment option that should be considered. ${ }^{54,55}$ Implantation concomitant with cataract surgery is currently the only Food and Drug 


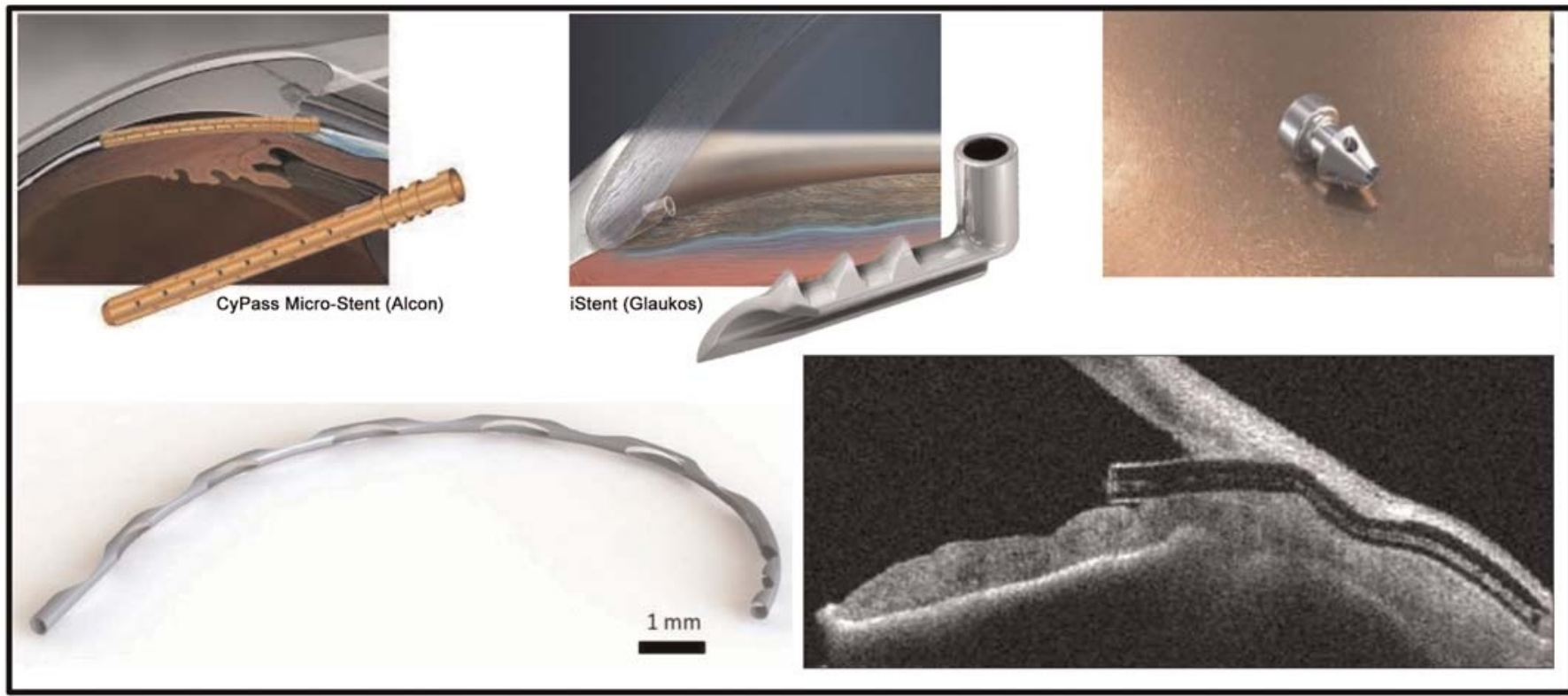

FIGURE 2. Minimally invasive glaucoma surgery (MIGS) devices. MIGS devices are devices that use an ab interno, microincisional approach, create minimal trauma to and disruption of normal anatomy and physiology, and show effective intraocular pressure lowering. MIGS devices should be straightforward, rapid, relatively easy, and appropriate for both glaucoma specialists and general ophthalmologists. Clockwise from top left: CyPass Micro-Stent, iStent Trabecular Micro-bypass Stent, iStent Inject, Hydrus Microstent, XEN Gel Stent.

Administration-approved indication for both the CyPass supraciliary stent (Alcon Laboratories) and the iStent trabecular stent (Glaukos, Inc., Laguna Hills, CA), although both may be used in standalone procedures in Europe. Cataract surgery alone provides intraocular pressure reduction of up to $15 \%$ in the first 12 to 24 months, but the effect seems to diminish over time, averaging $9 \%$ at 36 months. ${ }^{55}$ The Hydrus device currently in premarket approval evaluation is also for use with cataract surgery. The size of the patient population that could benefit from minimally invasive glaucoma surgery is quite large. In a recent retrospective chart review of all cataract surgeries performed in the outpatient setting at three locations in Colorado, approximately $18 \%$ of patients had comorbid glaucoma. ${ }^{56}$ Cataract surgery thus provides a unique opportunity to implant a minimally invasive glaucoma surgery device

TABLE 1. Minimally invasive glaucoma surgery devices

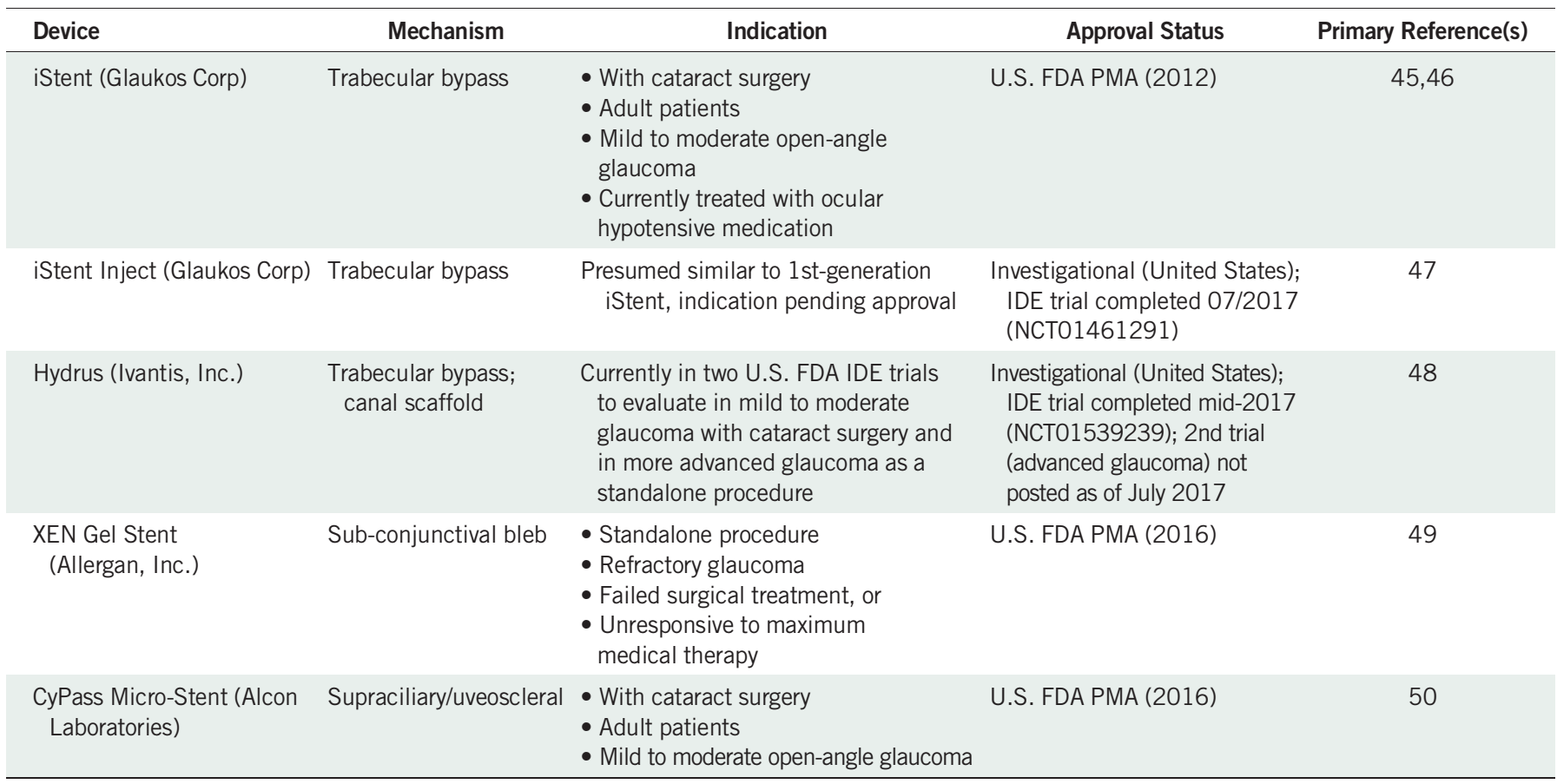

IDE = investigational device exemption; PMA = premarket approval; U.S. FDA = U.S. Food and Drug Administration. 
without the risk of a second surgery, providing the glaucoma patient with the potential for improved quality of life through medication reduction and intraocular pressure control less reliant on individual adherence.

Minimally invasive glaucoma surgery devices approved in the United States for the treatment of glaucoma increase outflow by three distinct mechanisms: subconjunctival outflow, trabecular bypass into Schlemm's canal, and uveoscleral flow into the suprachoroidal space.

\section{Subconjunctival Filtering Stent}

The XEN gel stent is indicated for refractory glaucoma in cases where surgical treatment has failed or in cases that are unresponsive to maximum tolerated medical therapy. ${ }^{49}$ XEN can be placed in phakic or pseudophakic eyes as a standalone procedure. XEN is also implanted with an ab interno surgical approach; however, the mechanism of action is more akin to filtration surgery than to the physiological outflow pathways (Schlemm's canal, uveoscleral). The XEN gel stent is a 6-mm-long tube with a 45- $\mu \mathrm{m}$ bore composed of porcine gelatin crosslinked with glutaraldehyde. It is placed in a scleral tunnel created by the beveled needle applicator and terminating just under the conjunctiva. Aqueous humor flows from the anterior chamber through the stent and forms a filtering bleb under the conjunctiva. The length of the stent coupled with the bore creates outflow resistance titrated to prevent hypotony. ${ }^{57}$ In the single-arm noncomparative trial supporting U.S. approval, $71 \%$ of patients achieved intraocular pressure reductions of $20 \%$ or greater from baseline on the same or fewer medications at 12 months of followup. The mean medication reduction was 1.8 (baseline, 3.5; 12 months, 1.7) for the 52 subjects (80\%) completing 12 months of follow-up. ${ }^{49}$ XEN implantation is less traumatic and tissue disruptive than traditional trabeculectomy. It was thought that this would result in less fibrosis and therefore decrease the need for bleb manipulations and needling. The rate of needling for XEN procedures is reported to be approximately $32 \%$ by 1 year postoperatively, similar to rates seen with conventional filtration procedures. ${ }^{49}$ Although XEN could conceivably be used with cataract surgery across the spectrum of glaucoma, the presence of a bleb and the potential for bleb complications and maintenance suggest that this device be reserved for more advanced cases. The somewhat more complicated surgical procedure (than for other minimally invasive glaucoma surgery) could limit adoption by cataract surgeons where the ideal concomitant procedure should add minimal time to the cataract extraction, be relatively easy procedurally, and require a minimum of follow-up.

\section{Trabecular Bypass Stents}

The iStent was the first minimally invasive glaucoma surgery device to receive a marketing authorization in the United States (in 2012). It is an L-shaped stent that is approximately $1.0 \mathrm{~mm}$ long and is made of heparin-coated titanium (Fig. 2). It has an internal bore of $120 \mu \mathrm{m}$. Properly placed, the long leg of the $L$ resides in Schlemm's canal with the short leg or snorkel protruding into the anterior chamber. There are numerous publications for the iStent; however, most are relatively small or case series or both and thus of relatively low-quality evidence. ${ }^{58}$ A randomized controlled trial with a sample size providing appropriate statistical power to test the hypothesis ( $N=240$ ) compared 1- and 2-year outcomes for the iStent + phacoemulsification versus phacoemulsification alone. ${ }^{45,46}$ At 1 year, $72 \%$ of patients randomized to the stent met the primary end point (intraocular pressure $\leq 21 \mathrm{mmHg}$ ) versus $50 \%$ for the control group. The difference between groups narrowed at 2 years ( $61 \%$ stent, $50 \%$ control). The proportion of patients achieving $20 \%$ reduction or greater from baseline intraocular pressure was $66 \%$ stent and $48 \%$ control at 1 year and $53 \%$ stent and $44 \%$ control at 2 years. Use of ocular hypotensive medications at 1 year postoperatively was reduced by an average of 1.4 (stent) or 1.1 (control) medications.

Several studies with the original iStent indicated that placement of a second or even third stent in the eye provided superior outcomes. ${ }^{59,60}$ This has been ascribed to a theoretical incremental increase in outflow facility with each additional stent. ${ }^{61}$ Alternatively, there may be a chance element involved in stent placement; Schlemm's canal is interrupted circumferentially by occasional septa, and not all collector channels are active; therefore, a second stent not only increases the potential volume of aqueous egress, but it also increases the likelihood of proximity to active collector channels. ${ }^{4,62}$ The second-generation trabecular bypass (iStent inject) from Glaukos has recently completed a randomized controlled trial to support a premarket approval submission (ClinicalTrials.gov identifier NCT01461291). Like the first generation, the new device is heparin-coated titanium but is much smaller $(360 \times 230 \mu \mathrm{m})$ with radial symmetry (Fig. 2) and is injected through the trabecular meshwork into Schlemm's canal. In contrast to the first generation, and presumably directed by findings from the multiple-stent studies cited above, it is intended that treatment consists of implantation of two stents. These are placed into the nasal aspect of Schlemm's canal at approximately two clock-hours apart. ${ }^{47}$ Results from the pivotal premarket approval trial are not yet public; however, one randomized controlled trial has been published enrolling 192 patients, randomized 1:1 to either two iStent inject stents or latanoprost/timolol fixed combination. ${ }^{47}$ At 12 months, the proportion of eyes with a $20 \%$ reduction or greater from baseline was 95\% for the stent group and $92 \%$ for the medication group. Mean intraocular pressure reductions at 12 months were 8.1 and $7.3 \mathrm{mmHg}$, respectively. ${ }^{47} \mathrm{~A}$ second small randomized controlled trial listed on ClinicalTrials.gov (NCT00721968) comparing iStent inject plus cataract surgery with cataract surgery alone was completed in 2012, although it has not been published; 18 (67\%) of 27 stent patients had intraocular pressure of $18 \mathrm{mmHg}$ or less at month 12 versus $24 \%$ for the cataract surgery-only group.

Although not yet approved in the United States, the Hydrus device (Ivantis, Inc., Irvine, CA) has recently completed a large premarket approval trial to obtain marketing authorization (ClinicalTrials.gov identifier NCT01539239). Hydrus is a Schlemm's canal scaffold composed of a nickel-titanium alloy. It is an 8-mm-long crescent tube with multiple windows along its length. The device is advanced into the canal leaving, 1 to $2 \mathrm{~mm}$ of the inlet segment in the anterior chamber. The device is intended to dilate and stent the canal facilitating aqueous outflow. While data are not yet available from the registration study, a smaller randomized controlled trial comparing Hydrus + cataract surgery with cataract surgery alone found that at 12 months $88 \%$ of Hydrus patients had at least a $20 \%$ reduction in intraocular pressure from baseline versus $74 \%$ for cataract surgery alone. ${ }^{48}$ The response rate was 80 and $46 \%$, respectively, at 24 months. Mean intraocular pressure reductions from baseline at 12 months were 9.7 (Hydrus) and $9.2 \mathrm{mmHg}$ (cataract surgery only). However, at 24 months, these were 9.4 (Hydrus) and $7.4 \mathrm{mmHg}$ (cataract surgery only). Medication reduction was essentially similar to that reported for iStent (above) with an average decrease from baseline of 1.5 medications at both 12 and 24 months. ${ }^{48}$ 
The safety profile for the trabecular bypass devices seems to be very good, although comparisons across studies and with other minimally invasive glaucoma surgery interventions should be made with caution because of variability in the adverse event collection methodology. ${ }^{45}$ Most of the adverse events reported are those that would be expected for cataract surgery whether or not a stent was placed. Notably, best corrected visual acuities measured at follow-up visits 1 month and greater postoperatively were not different between stent and cataract surgery-only groups. ${ }^{48}$ In the Hydrus study, it was reported that peripheral anterior synechiae formed at or near the inlet of the device in $12 \%$ of patients but did not affect efficacy of the device. Hyphema or blood reflux into the anterior chamber has been reported at a high incidence $(>90 \%)$ in some iStent studies ${ }^{63,64}$ and not reported at all in others. ${ }^{45-47,60,65}$

\section{Supraciliary Stents}

The second major physiological route for aqueous outflow is the uveoscleral route. Indeed, given that pathologic changes to the trabecular pathway are acknowledged to be the proximal cause of elevated intraocular pressure in open-angle glaucoma, the uveoscleral pathway has been exploited both pharmacologically (i.e., prostaglandin analogs) and surgically (e.g., cyclodialysis; ab externo shunts) as an independent means of lowering intraocular pressure. The supraciliary space is a virtual space lying between the ciliary body and the sclera. Posteriorly, it is continuous with the suprachoroidal space. Supraciliary stents are placed in the anterior chamber angle with the device and applicator, making a blunt dissection between the scleral spur and the iris/ciliary body. A supraciliary stent essentially provides a controlled cyclodialysis. With proper insertion, the size of the cleft is dictated by the dimensions of the stent, which greatly minimizes the risks of uncontrolled hypotony or bleeding. Placement of the stent provides a means of maintaining the cleft and allowing continued outflow.

A supraciliary ab interno approach to enhance uveoscleral flow offers several advantages over other methods. In comparison to trabecular bypasses, successful placement is not dependent on local variability such as proximity to active collector channels. In addition, flow directed through Schlemm's canal and out the collector channels is limited by episcleral venous pressure, typically 10 to $11 \mathrm{mmHg}$; uveoscleral flow instead egresses through the sclera into the lymphatics and the vortex system and thus is not similarly limited. ${ }^{66-68}$ Hypothetically, because outflow via the uveoscleral pathway is not linked to episcleral venous pressure, which is higher at night when in the supine position, a supraciliary stent might provide better ability to blunt nocturnal intraocular pressure rises. ${ }^{69}$ Unlike a filtering procedure such as the XEN implant, there is no conjunctival bleb subject to scarring and other postoperative issues. The ab interno implantation is in contrast to more invasive ab externo implanted suprachoroidal devices such as the SOLX Gold (SOLX, Inc., Waltham, MA). This less traumatic approach should result in less inflammation, fibrosis, and device failure, although long-term follow-up data are required before definitive conclusions can be drawn. ${ }^{68}$

Currently, the only commercially available supraciliary stent is the CyPass Micro-Stent. A second device, the iStent Supra, is currently in an Investigational Device Exemption clinical trial (NCT01461278) to support U.S. Food and Drug Administration approval. CyPass has been available in Europe since 2008. Marketing approval in the United States (2016) was based on the COMPASS trial, at the time the largest randomized clinical trial comparing a minimally invasive glaucoma surgery device with cataract surgery to cataract surgery alone. In COMPASS, 505 patients were randomized in a 3:1 allocation ratio to CyPass with phacoemulsification or to phacoemulsification alone. One and 2 years after surgery, 82 and $77 \%$ of the CyPass group had achieved a $20 \%$ reduction in intraocular pressure from baseline compared with 66 and $60 \%$ for phacoemulsification alone. Mean intraocular pressure reductions from baseline were 7.9 and $6.2 \mathrm{mmHg}$ at 1 year and 7.4 and $5.4 \mathrm{mmHg}$ at 2 years for the CyPass and phacoemulsification groups, respectively, showing that the intraocular pressure-lowering effect of the stent was sustained over this interval. ${ }^{50}$ Elimination of medications or simplification of dosing regimen is a critical measure of an intervention such as CyPass that is relevant to improving adherence. In COMPASS, the mean numbers of medications at baseline were 1.4 for CyPass and 1.3 for phacoemulsification only. By 2 years, these were 0.2 for CyPass and 0.6 for the control group. The number of patients on no medications at 2 years was $85 \%$ for CyPass and $59 \%$ for the phacoemulsification control compared with baseline of 17 and $20 \%{ }^{50}$ Similarly, Hoh showed that in a cohort of patients controlled at baseline on two medications following CyPass implantation (with or without cataract surgery), an average of 1.5 medications were discontinued at 12 months and 0.9 medication through 3 years while maintaining intraocular pressure control at baseline levels. ${ }^{70,71}$

Similar to the trabecular stents, adverse events for CyPass attributable to the device or procedure were infrequent and nonserious. ${ }^{50}$ The most common event was best corrected visual acuity loss of 10 or more letters, which occurred almost twice as frequently in the phacoemulsification-only group than for CyPass (15.3 vs. $8.8 \%$ ) and was transient, with more than $98 \%$ in both groups having best corrected visual acuity better than $20 / 40$ by the end of the study. Other adverse events were iritis (7.8\%), intraocular pressure elevation (4.0\%), and corneal edema (3.2\%); all were transient, and incidences were not statistically significantly different between stent and control groups. ${ }^{50}$ There were 11 adverse events (2.9\%) for numerical hypotony (pressure at or $<6 \mathrm{mmHg}$ ), which was, again, transient, did not affect functional outcomes such as visual acuity, and resolved without sequela (e.g., maculopathy, choroidal effusion)..$^{50}$

\section{LIMITATIONS}

The present work is not intended to be a comprehensive and systematic review of all currently and historically available treatments for elevated intraocular pressure. It is instead focused primarily on minimally invasive glaucoma surgery. Earlier treatment modalities and other approaches aimed at accomplishing similar ends, that is, reduction in the medication burden and as a consequence better adherence, are also discussed in order to place minimally invasive glaucoma surgery in the proper historical context. Treatments reserved for advanced and refractory glaucoma such as filtration surgery, tubes and shunts (e.g., Ahmed Valve), and cyclodestructive techniques are therefore out of scope. There are also limitations in the published literature on minimally invasive glaucoma surgery. Follow-up providing safety and effectiveness data beyond 2 years is generally not available. This is because the devices are relatively new, and the longer-term data are still being collected in postmarket studies. Data on 3-, 4-, and 5-year outcomes should be forthcoming in the future. Head-to-head comparisons of effectiveness and safety are also missing from the literature with a few exceptions. ${ }^{72}$ This gap will also be filled as ongoing studies are 
completed and results published (e.g., iStent and Hydrus study, ClinicalTrials.gov identifier NCT02024464). The potential to use devices with different mechanisms of action is yet to be explored as is, with some exceptions, the utility of these devices in more refractory cases. ${ }^{70}$

\section{CONCLUSIONS}

The vision and mission of the World Council of Optometry include the following statement: "Our objectives... the worldwide improvement and conservation of human vision."73 Two decades ago, Spaeth ${ }^{74}$ opined that noncompliance was not so much a patient problem as it was indicative of poor understanding and communication between doctor and patient. As the point personnel in the effort for improvement and conservation of vision, optometrists are uniquely positioned to take a holistic view of the glaucoma patient including emotional, physical, and social characteristics potentially affecting the patient's ability to adhere to treatment. Patient adherence is the wild card in the deck for controlling glaucoma progression. Patients with glaucoma tend to be older and have systemic comorbidities that can affect ability to adhere to treatment, but there may be non-age-dependent psychosocial factors as well. ${ }^{74}$
The burden of medication not only includes cost and unwanted adverse effects, but also demands that the patient take them as directed. Older individuals may have difficulties with instillation, and if arthritic, simply removing caps and squeezing drops can be challenging. They may have memory loss. They may be taking multiple other medications. The barriers to good compliance are many and significant. Innovations ranging from drug depots and implants, to laser technology, to minimally invasive glaucoma surgery, are all directed at addressing this very real gap between prescribed treatment and that which is actually delivered. Minimally invasive glaucoma surgery has been shown to provide a safe surgical approach, particularly in combination with cataract surgery, and may provide good intraocular pressure-lowering effectiveness and the opportunity to reduce or eliminate ocular hypotensive medications. Identifying those patients who could benefit from minimally invasive glaucoma surgery through decreased dependence on medications and the potential for better control of their glaucoma is in alignment with the overall goals of optometric practice. In the future, minimally invasive glaucoma surgery devices may find utility beyond mild to moderate glaucoma and in conjunction with cataract surgery. Further studies for many of these devices aimed at expanding the indications are underway including more severe glaucoma and as standalone procedures.

\section{ARTICLE INFORMATION}

Submitted: August 4, 2017

Accepted: December 1, 2017

Funding/Support: None of the authors have reported funding/support.

Conflict of Interest Disclosure: Jaime E. Dickerson is an employee of Alcon.

Author Contributions: Conceptualization: MF, JED; Resources: MF, JED; Writing - Original Draft: MF, JED; Writing - Review \& Editing: MF, JED; Data Curation: JED; Formal Analysis: JED; Supervision: JED.

\section{REFERENCES}

1. Tham YC, Li X, Wong TY, et al. Global Prevalence of Glaucoma and Projections of Glaucoma Burden through 2040: A Systematic Review and Meta-analysis. Ophthalmology 2014;121:2081-90.

2. Kerrigan-Baumrind LA, Quigley HA, Pease ME, et al. Number of Ganglion Cells in Glaucoma Eyes Compared with Threshold Visual Field Tests in the Same Persons. Invest Ophthalmol Vis Sci 2000;41:741-8.

3. Quigley HA, Dunkelberger GR, Green WR. Retinal Ganglion Cell Atrophy Correlated with Automated Perimetry in Human Eyes with Glaucoma. Am J Ophthalmol 1989;107:453-64.

4. Allingham RR, Damji K, Freedman $S$, et al. Shields Textbook of Glaucoma, 5th ed. Philadelphia, PA Lippincott Williams \& Wilkins; 2005.

5. Kass MA, Heuer DK, Higginbotham EJ, et al. The Ocular Hypertension Treatment Study: A Randomized Tria Determines That Topical Ocular Hypotensive Medication Delays or Prevents the Onset of Primary Openangle Glaucoma. Arch Ophthalmol 2002;120:70113; discussion 829-30.
6. Leske MC, Heijl A, Hussein M, et al. Factors for Glaucoma Progression and the Effect of Treatment: The Early Manifest Glaucoma Trial. Arch Ophthalmol 2003;121: 48-56.

7. The Advanced Glaucoma Intervention Study (AGIS): The Relationship between Control of Intraocular Pressure and Visual Field Deterioration. The AGIS Investigators. Am J Ophthalmol 2000;130:429-40.

8. Dul MW. The Glaucomas. In: Bartlett JD, Jaanus SD, eds. Clinical Ocular Pharmacology. 5th ed. St. Louis, MO: Butterworth Heinemann/Elsevier; 2008; Chapter 34.

9. Krieglstein GK. Medical Treatment of Glaucoma. In: Hitchings RA, ed. Glaucoma, London: BMJ; 2000;77-84.

10. European Glaucoma Society Terminology and Guidelines for Glaucoma, 4th ed. - Chapter 3: Treatment Principles and Options Supported by the EGS Foundation. Br J Ophthalmol 2017;101:130-95.

11. Fingeret M, Mancil L, Bailey IL, et al. American Optometric Association: Optometric Clinical Practice Guideline: Care of the Patient with Open Angle Glaucoma; 2011. Available at: https://www.aoa.org/documents/ optometrists/CPG-9.pdf. Accessed December 2, 2017.

12. Fingeret $M$. The Optometrist's Role in Glaucoma Care. Glaucoma Today 2014; May/June 2014:30,41. Available at: http://glaucomatoday.com/2014/06/ the-optometrists-role-in-glaucoma-care/. Accessed December 2, 2017.

13. Francis BA, Singh K, Lin SC, et al. Novel Glaucoma Procedures: A Report by the American Academy of Ophthalmology. Ophthalmology 2011;118:1466-80.

14. Vinod K, Gedde SJ. Clinical Investigation of New Glaucoma Procedures. Curr Opin Ophthalmol 2017;28: 187-93.

15. Viswanathan M, Golin CE, Jones CD, et al. Interventions to Improve Adherence to Self-administered Medications for Chronic Diseases in the United States: A Systematic Review. Ann Intern Med 2012;157: 785-95.
16. Olthoff CM, Schouten JS, van de Borne BW, et al. Noncompliance with Ocular Hypotensive Treatment in Patients with Glaucoma or Ocular Hypertension. An Evidence-based Review. Ophthalmology 2005;112: 953-61.

17. Broadway DC, Cate H. Pharmacotherapy and Adherence Issues in Treating Elderly Patients with Glaucoma. Drugs Aging 2015;32:569-81.

18. Horsley MB, Wilson MR. Epidemiology of Glaucoma. In: Kahook MY, Schuman JS, eds. Chandler and Grant's Glaucoma, 5th ed. Thorofare, NJ: Slack; 2013.

19. Friedman DS, Hahn SR, Gelb L, et al. Doctor-patient Communication, Health-related Beliefs, and Adherence in Glaucoma Results from the Glaucoma Adherence and Persistency Study. Ophthalmology 2008;115:1320-7 e1-3.

20. Sleath B, Blalock SJ, Stone JL, et al. Validation of a Short Version of the Glaucoma Medication Self-efficacy Questionnaire. Br J Ophthalmol 2012;96:258-62.

21. Stryker JE, Beck AD, Primo SA, et al. An Exploratory Study of Factors Influencing Glaucoma Treatment Adherence. J Glaucoma 2010;19:66-72.

22. Lacey J, Cate H, Broadway DC. Barriers to Adherence with Glaucoma Medications: A Qualitative Research Study. Eye (Lond) 2009;23:924-32.

23. Hall E, Lee SY, Clark PC, et al. Social Ecology of Adherence to Hypertension Treatment in Latino Migrant and Seasonal Farmworkers. J Transcult Nurs 2016;27:33-41.

24. Newman-Casey PA, Robin AL, Blachley T, et al. The Most Common Barriers to Glaucoma Medication Adherence: A Cross-sectional Survey. Ophthalmology 2015; 122:1308-16.

25. Hennessy AL, Katz J, Covert D, et al. A Video Study of Drop Instillation in Both Glaucoma and Retina Patients with Visual Impairment. Am J Ophthalmol 2011;152: 982-8.

26. Sleath B, Blalock S, Covert D, et al. The Relationship between Glaucoma Medication Adherence, Eye 
Drop Technique, and Visual Field Defect Severity. Ophthalmology 2011;118:2398-402.

27. Higginbotham EJ. Considerations in Glaucoma Therapy: Fixed Combinations Versus Their Component Medications. Clin Ophthalmol 2010;4:1-9.

28. Barnebey HS, Robin AL. Adherence to Fixed Combination Versus Unfixed Travoprost 0.004\%/Timolol 0.5\% for Glaucoma or Ocular Hypertension: A Randomized Trial. Am J Ophthalmol 2017;176:61-9.

29. Urquhart J. The Electronic Medication Event Monitor. Clin Pharmacokinet 1997;32:345-56.

30. Ho LY, Camejo L, Kahook MY, et al. Effect of Audible and Visual Reminders on Adherence in Glaucoma Patients Using a Commercially Available Dosing Aid. Clin Ophthalmol 2008;2:769-72.

31. Ozdemir S, Wong TT, Allingham RR, et al. Predicted Patient Demand for a New Delivery System for Glaucoma Medicine. Medicine (Baltimore) 2017;96:e6626.

32. Lavik E, Kuehn MH, Kwon YH. Novel Drug Delivery Systems for Glaucoma. Eye (Lond) 2011;25:578-86.

33. Quigley HA, Pollack IP, Harbin TS, Jr. Pilocarpine Ocuserts. Long-term Clinical Trials and Selected Pharmacodynamics. Arch Ophthalmol 1975;93:771-5.

34. Linden C, Alm A. Latanoprost Twice Daily Is Less Effective Than Once Daily: Indication of Receptor Subsensitivity? Curr Eye Res 1998;17:567-72.

35. Robin AL, Clark AF, Covert DW, et al. Anterior Juxtascleral Delivery of Anecortave Acetate in Eyes with Primary Open-angle Glaucoma: A Pilot Investigation. Am J Ophthalmol 2009;147:45-50 e2.

36. Aref AA. Sustained Drug Delivery for Glaucoma: Current Data and Future Trends. Curr Opin Ophthalmol 2017;28:169-74.

37. Prum BE, Jr., Rosenberg LF, Gedde SJ, et al. Primary Open-angle Glaucoma Preferred Practice Pattern $®$ Guidelines. Ophthalmology 2016;123:P41-111.

38. Francis BA, Loewen N, Hong B, et al. Repeatability of Selective Laser Trabeculoplasty for Open-angle Glaucoma. BMC Ophthalmol 2016;16:128.

39. Polat J, Grantham L, Mitchell K, et al. Repeatability of Selective Laser Trabeculoplasty. Br J Ophthalmol 2016;100: 1437-41.

40. Martinez-de-la-Casa JM, Garcia-Feijoo J, Castillo A, et al. Selective Vs. Argon Laser Trabeculoplasty: Hypotensive Efficacy, Anterior Chamber Inflammation, and Postoperative Pain. Eye (Lond) 2004;18:498-502.

41. Latina MA, Sibayan SA, Shin DH, et al. Q-switched 532-Nm Nd:YAG Laser Trabeculoplasty (Selective Laser Trabeculoplasty): A Multicenter, Pilot, Clinical Study. Ophthalmology 1998;105:2082-8.

42. Juzych MS, Chopra V, Banitt MR, et al. Comparison of Long-term Outcomes of Selective Laser Trabeculoplasty Versus Argon Laser Trabeculoplasty in Open-angle Glaucoma. Ophthalmology 2004;111:1853-9.

43. Hirn C, Zweifel SA, Töteberg-Harms M, et al. [Effectiveness of Selective Laser Trabeculoplasty in Patients with Insufficient Control of Intraocular Pressure Despite Maximum Tolerated Medical Therapy]. Ophthalmologe 2012;109:683-90.

44. Caprioli J, Kim JH, Friedman DS, et al. Special Commentary: Supporting Innovation for Safe and Effective
Minimally Invasive Glaucoma Surgery: Summary of a Joint Meeting of the American Glaucoma Society and the Food and Drug Administration, Washington, DC, February 26, 2014. Ophthalmology 2015;122: 1795-801.

45. Samuelson TW, Katz LJ, Wells JM, et al. Randomized Evaluation of the Trabecular Micro-bypass Stent with Phacoemulsification in Patients with Glaucoma and Cataract. Ophthalmology 2011;118:459-67.

46. Craven ER, Katz LJ, Wells JM, et al. Cataract Surgery with Trabecular Micro-bypass Stent Implantation in Patients with Mild-to-Moderate Open-angle Glaucoma and Cataract: Two-year Follow-up. J Cataract Refract Surg 2012;38:1339-45.

47. Fea AM, Belda JI, Rekas M, et al. Prospective Unmasked Randomized Evaluation of the iStent Inject ${ }^{\circledR}$ Versus Two Ocular Hypotensive Agents in Patients with Primary Open-angle Glaucoma. Clin Ophthalmol 2014; 8:875-82.

48. Pfeiffer N, Garcia-Feijoo J, Martinez-de-la-Casa JM, et al. A Randomized Trial of a Schlemm's Canal Microstent with Phacoemulsification for Reducing Intraocular Pressure in Open-angle Glaucoma. Ophthalmology 2015; 122:1283-93.

49. Allergan, Inc. XEN Gel Stent (Instructions for Use), Irvine, CA: Allergan, Inc.; 2017.

50. Vold S, Ahmed II, Craven ER, et al. Two-year COMPASS Trial Results: Supraciliary Microstenting with Phacoemulsification in Patients with Open-Angle Glaucoma and Cataracts. Ophthalmology 2016;123: 2103-12.

51. Ahmed K II. MIGS and the FDA: What's in a Name? Ophthalmology 2015;122:1737-9.

52. Saheb H, Ahmed K II. Micro-invasive Glaucoma Surgery: Current Perspectives and Future Directions. Curr Opin Ophthalmol 2012;23:96-104.

53. Khaimi MA, Reyes M. Filtering Surgery in the Management of Glaucoma. In: Kahook MY, Schuman JS, eds. Chandler and Grant's Glaucoma. 5th ed. Thorofare, NJ: Slack; 2013: Chapter 59

54. Richter GM, Coleman AL. Minimally Invasive Glaucoma Surgery: Current Status and Future Prospects. Clin Ophthalmol 2016;10:189-206.

55. Armstrong JJ, Wasiuta T, Kiatos E, et al. The Effects of Phacoemulsification on Intraocular Pressure and Topical Medication Use in Patients with Glaucoma: A Systematic Review and Meta-analysis of 3-Year Data. J Glaucoma 2017;26:511-22.

56. Ianchulev T, Litoff D, Ellinger D, et al. Office-based Cataract Surgery: Population Health Outcomes Study of More Than 21000 Cases in the United States. Ophthalmology 2016;123:723-8.

57. Lewis RA. Ab Interno Approach to the Subconjunctival Space Using a Collagen Glaucoma Stent. J Cataract Refract Surg 2014;40:1301-6.

58. Zhang ML, Hirunyachote $P$, Jampel H. Combined Surgery Versus Cataract Surgery Alone for Eyes with Cataract and Glaucoma. Cochrane Database Syst Rev 2015:CD008671.

59. Belovay GW, Naqi A, Chan BJ, et al. Using Multiple Trabecular Micro-bypass Stents in Cataract Patients to
Treat Open-angle Glaucoma. J Cataract Refract Surg 2012;38:1911-7

60. Katz LJ, Erb C, Carceller GA, et al. Prospective, Randomized Study of One, Two, or Three Trabecular Bypass Stents in Open-angle Glaucoma Subjects on Topical Hypotensive Medication. Clin Ophthalmol 2015;9: 2313-20.

61. Hunter KS, Fjield T, Heitzmann H, et al. Characterization of Micro-invasive Trabecular Bypass Stents by Ex Vivo Perfusion and Computational Flow Modeling. Clin Ophthalmol 2014;8:499-506.

62. Huang AS, Camp A, Xu BY, et al. Aqueous Angiography: Aqueous Humor Outflow Imaging in Live Human Subjects. Ophthalmol 2017;124:1249-51.

63. Klamann MK, Gonnermann J, Pahlitzsch M, et al. Istent Inject in Phakic Open Angle Glaucoma. Graefes Arch Clin Exp Ophthalmol 2015;253:941-7.

64. Gonnermann J, Bertelmann E, Pahlitzsch M, et al. Contralateral Eye Comparison in MICS \& MIGS: Trabectome $®$ vs. iStent inject $®$. Graefes Arch Clin Exp Ophthalmol 2017;255:359-65.

65. Berdahl J, Voskanyan L, Myers JS, et al. Implantation of Two Second-generation Trabecular Microbypass Stents and Topical Travoprost in Open-angle Glaucoma Not Controlled on Two Preoperative Medications: 18-Month Follow-up. Clin Exp Ophthalmol 2017;45:797-802.

66. Kerr NM, Wang J, Barton K. Minimally Invasive Glaucoma Surgery as Primary Stand-alone Surgery for Glaucoma. Clin Exp Ophthalmol 2017;45:393-400.

67. Figus $M$, Posarelli $C$, Passani $A$, et al. The Supraciliary Space as a Suitable Pathway for Glaucoma Surgery: Ho-hum or Home Run? Surv Ophthalmol 2017; 62:828-37.

68. Bailey AK, Sarkisian SR, Jr., Vold SD. Ab Interno Approach to the Suprachoroidal Space. J Cataract Refract Surg 2014;40:1291-4.

69. Sultan M, Blondeau P. Episcleral Venous Pressure in Younger and Older Subjects in the Sitting and Supine Positions. J Glaucoma 2003;12:370-3.

70. Hoeh H, Vold SD, Ahmed IK, et al. Initial Clinical Experience with the CyPass Micro-Stent: Safety and Surgical Outcomes of a Novel Supraciliary Microstent. J Glaucoma 2016;25:106-12.

71. Hoh H, Rau M. Three-year Results from the Cycle Study for Supraciliary Micro-stent Implantation Combined with Cataract Surgery for Open-angle Glaucoma. Paper presented at the 2017 ASCRS-ASOA Symposium \& Congress; May 5-9, 2017; Los Angeles, CA. Available at: https://ascrs.confex.com/ascrs/17am/meetingapp. cgi/Paper/3256. Accessed December 2, 2017.

72. Fea AM, Ahmed II, Lavia C, et al. Hydrus Microstent Compared to Selective Laser Trabeculoplasty in Primary Open Angle Glaucoma: One Year Results. Clin Exp Ophthalmol 2017;45:120-7.

73. World Council of Optometry. Vision and Mission; 2017. Available at: http://worldcouncilofoptometry. info/about-us/vision-and-mission/. Accessed December 2, 2017.

74. Spaeth GL. "Noncompliance"-Not a Patient Problem, But Rather a Sign of a Sick Doctor-patient Relationship. Ophthalmic Surg 1995;26:189-93. 